See discussions, stats, and author profiles for this publication at: https://www.researchgate.net/publication/335927068

Childhood adversity and psychosis in detained inpatients from medium to high secured units: Results from the Scottish census survey

Article in Child Abuse \& Neglect · July 2019

DOI: 10.1016/j.chiabu.2019.104094

\section{CITATIONS}

6 authors, including:

Thanos Karatzias

Edinburgh Napier University

181 PUBLICATIONS 2,834 CITATIONS

SEE PROFILE

Jamie Pitcairn

National Health Service

7 PUBLICATIONS 136 CITATIONS

SEE PROFILE

Some of the authors of this publication are also working on these related projects:

Local Area Coordinators in Scotland View project

Mental Health of Prisoners View project

READS

236

Mark Shevlin

Ulster University

415 PUBLICATIONS 9,873 CITATIONS

SEE PROFILE

Lindsay Thomson

The University of Edinburgh

111 PUBLICATIONS 660 CITATIONS

SEE PROFILE 
Research article

\title{
Childhood adversity and psychosis in detained inpatients from medium to high secured units: Results from the Scottish census survey
}

\author{
Thanos Karatzias ${ }^{\mathrm{a}, \mathrm{b}, *}$, Mark Shevlin ${ }^{\mathrm{c}}$, Jamie Pitcairn ${ }^{\mathrm{d}}$, Lindsay Thomson ${ }^{\mathrm{e}}$, \\ Adam Mahoney $^{\mathrm{f}}$, Philip Hyland ${ }^{\mathrm{g}}$ \\ ${ }^{a}$ Edinburgh Napier University, School of Health \& Social Care, Edinburgh, UK \\ ${ }^{\mathrm{b}}$ NHS Lothian, Rivers Centre for Traumatic Stress, Edinburgh, UK \\ ${ }^{\mathrm{c}}$ Ulster University, School of Psychology, Derry, UK \\ ${ }^{\mathrm{d}}$ NHS Scotland, The State Hospital \& Forensic Network, UK \\ ${ }^{\mathrm{e}}$ Edinburgh University, Division of Psychiatry, Edinburgh, UK \\ ${ }^{\mathrm{f}}$ HMP YOI Cornton Vale, Scottish Prison Service, Stirling, UK \\ ${ }^{\mathrm{g}}$ National College of Ireland, School of Business, Dublin, Ireland
}

\section{A R T I C L E I N F O}

\section{Keywords:}

Childhood adversity

Psychosis

Forensic patients

\begin{abstract}
A B S T R A C T
Background: There is limited data available regarding the most common forms of psychiatric illness, the occurrence of childhood adversity, and the link between childhood adversity and criminal and psychiatric outcomes amongst forensic inpatients.

Aims: Using census data for all Scottish forensic inpatients, we investigated the most common primary psychiatric diagnoses in forensic settings, the occurrence of childhood adversity amongst forensic inpatients, and whether childhood adversity experiences significantly predict a range of criminal and psychiatric outcomes.

Method: Data for the current study were drawn from 'The Scottish Forensic Network Inpatient Census' $(\mathrm{N}=422)$. The Responsible Medical Officers and other members of the clinical team collected all data from official patient records. All forensic inpatients across high, medium, and low security sites were surveyed.

Results: The majority of patients had a psychotic disorder as their primary diagnosis (86.4\%), with schizophrenia being the most common (70.0\%). Childhood adversity was highly prevalent (79.2\%), with physical abuse being the most common adverse experience $(40.1 \%)$. Increased levels of childhood adversity were significantly associated with an increased risk of criminal convictions, self-reported abuse of animals, suicidal and self-injurious behaviour, and problematic use of drugs or alcohol.

Conclusions: Considering the association between adversity and psychosis, trauma informed care is essential for the mental health and forensic needs of this population.
\end{abstract}

\section{Introduction}

Exposure to adversity during childhood is a well-established risk factor for diagnosis of psychiatric disorders (e.g. Bentall,

\footnotetext{
* Corresponding author at: Edinburgh Napier University, Sighthill Campus, Sighthill Court, Edinburgh EH11 4BN, Scotland, UK.

E-mail address: t.karatzias@napier.ac.uk (T. Karatzias).
} 
Wickham, Shevlin, \& Varese, 2012; Jonas et al., 2011; Shevlin, Dorahy, \& Adamson, 2007), engagement in criminal behaviour (Avery, Hutchinson, \& Whitaker, 2002; Basto-Pereira, Miranda, Ribeiro, \& Maia, 2016), and the seriousness of criminal behaviour (Jones, 2015; Karatzias et al., 2018). These associations have also been increasingly well described in female offending populations (de Vogel \& Nicholls, 2016). A history of adversity in childhood has also been observed amongst forensic inpatients (Spitzer, Chevalier, Gillner, Freyberger, \& Barlow, 2006) and these experiences have been associated with an increased risk of suicidal behaviour in this population group (Dudeck et al., 2016).

The prevalence of psychiatric disorders has been reported in both remand prisoners (26\% had one or more disorders; Birmingham, Mason, \& Grubin, 1996) and the general prison population (50\% screened positive for a personality disorder, and $66 \%$ screened positive for any neurotic disorder; Singleton, Meltzer, Gatward, Coid, \& Deasy, 1998). Existing data also suggest that psychotic disorders and substance abuse disorders are common in this population group (Corbett et al., 2018; Kivimies, RepoTiihonen, \& Tiihonen, 2012). A meta-analysis of 33,588 prisoners from 24 countries found that $3.6 \%$ and $3.9 \%$ of male and female prisoners, respectively, suffered from a psychotic illness, and $10.2 \%$ and $14.1 \%$ of male and female prisoners, respectively, suffered from major depression (Fazel \& Seewald, 2012). However, the co-occurrence of childhood adversity and psychosis is not well understood in detained patients. In the current study, census data collected from all forensic inpatients in Scotland was used to determine: (1) The most frequently occurring primary psychiatric diagnosis; (2) the frequency and types of childhood adversity experiences that characterise this population, and whether these experiences vary according to gender; and (3) if childhood adversity experiences significantly predict multiple criminal (criminal convictions, and abuse of animals) and psychiatric (psychiatric admissions, suicidal and self-injurious behaviour, and problematic use of drugs and alcohol) outcomes whilst controlling for age, gender, and educational status.

\section{Methods}

\subsection{Procedure and participants}

Data were drawn from 'The Scottish Forensic Network Inpatient Census'. The Forensic Network Census, as part of the subsequent and ongoing Forensic Network Inpatient database, sought ethical approval as a Research Database. Ethical approval for the Research Database was granted by Scotland A REC in August 2013. In addition to ethical approval, data supply agreements were put in place for every health board area participating in the annual Census prior to the first data collection point in November 2013. The census employed a point-prevalence methodology and was based on all 23 Scottish Forensic Managed Care Network inpatient sites in Scotland. Each site was given detailed criteria to assist in defining which patients should be considered for the purpose of the census, and the patient data was completed in November 2013. The coordinating census team sent out data collection sheets to all Responsible Medical Officers (RMO) for the forensic inpatients under their care, and these forms were completed by the RMO and their senior medical trainees with support from other members of the clinical team. Forms were returned to the census team using a pre-determined secure transfer process. The census team and research assistants also offered support and advice to all participating sites to ensure consistent completion. The data were collated from existing data sources, primarily patient notes.

The inclusion criteria for the census were: All inpatients within High and Medium secure establishments across the Forensic Managed Care Network. In addition, forensic inpatients were identified from low secure units, rehab units, and intensive psychiatric care units (IPCUs) using the definition of mentally disordered offenders. The Scottish Office policy on Health, Social Work and Related Services for Mentally Disordered Offenders in Scotland describes mentally disordered offenders as those who are:

"Considered to suffer from a mental disorder as defined in the Mental Health (Care and Treatment) (Scotland) Act 2003, whether or not they are, or may be, managed under its provisions and come to the attention of the criminal justice system or whose behaviour poses a risk of such contact" (Scottish Office, 1999 - with update for 2003 Act).

This includes everyone currently being treated and detained under a criminal section of mental health legislation, namely Assessment Orders, Treatment Orders, Compulsion Orders, Interim-Compulsion Orders, Restriction Orders, Hospital Directions, Transferred Prisoners, and Temporary Hospital Orders. Patients were also included in the census if they had been directly transferred from high or medium security services, were detained under compulsory treatment orders, were previously subject to criminal section under the mental health legislation, or had an identified bed in an in-patient setting, regardless of suspension of detention.

In total, 422 forensic inpatients were included in the census. The majority were male $(91.0 \%, \mathrm{n}=384)$, and British (95.3\%, $\mathrm{n}=402)$. The mean age of the population was 41.47 years $(M d n=41.00, \mathrm{SD}=11.83$, Range $=18-76$ years). The majority of individuals were single $(78.0 \%, \mathrm{n}=329)$, and approximately one-third had children $(33.4 \%, \mathrm{n}=137)$. More than half of all forensic inpatients did not finish school $(57.3 \%, \mathrm{n}=197)$.

\subsection{Measures}

\subsubsection{Childhood trauma and adversity experiences}

Nineteen adversity experiences (see Table 2 for a full list) that occurred during a person's first 18 years of life were recorded on a 'Yes' (1) or 'No' (0) basis. A total score of childhood adversity was calculated ranging from 0-19.

\subsubsection{Prior criminal convictions}

The majority of inpatients possessed a history of at least one prior criminal conviction $(80.0 \%, \mathrm{n}=324)$. 


\subsubsection{History of animal abuse}

A small number of inpatients had a history of engagement in abuse of animals $(6.3 \%, \mathrm{n}=19)$.

\subsubsection{Prior psychiatric admissions}

The number of prior psychiatric admissions was recorded for each participant. A categorical variable was created reflecting those who had no prior psychiatric admissions, and those who had at least one prior psychiatric admission. The majority of inpatients had been previously admitted to a psychiatric setting $(82.0 \%, \mathrm{n}=260)$.

\subsubsection{Suicidal behaviour and self-injurious behaviour}

History of suicidal and self-injurious behaviour was recorded on a 'Yes' (1) or 'No' (0) basis. Approximately two-thirds possessed a history of suicidal and self-injurious behaviour $(66.2 \%, \mathrm{n}=261)$.

\subsubsection{Problematic drug and alcohol usage}

History of problematic (i) non-intravenous drug use, (ii) intravenous drug use, and (iii) alcohol use was recorded. If a respondent screened positive for any of these, they were defined as having a problem with drugs or alcohol. The majority of respondents screened positive for problematic use of drugs or alcohol $(86.4 \%, \mathrm{n}=368)$.

\subsection{Analytic plan}

Descriptive statistics were used to describe the most common primary psychiatric diagnosis, and the frequency of childhood adversity. Pearson chi-square tests were conducted to test for gender differences in the occurrence of each type of childhood adversity, and an independent samples $t$-test was conducted to determine mean differences in total adversity for males and females. An initial set of five binary logistic regression analyses were conducted to determine if childhood adversity predicted each criminal behaviour related outcome (prior criminal convictions, history of animal abuse) and each psychiatric related outcome (prior psychiatric admissions, history of suicidal and self-injurious behaviour, and problematic drug or alcohol use) whilst controlling for age, gender $(0=$ Female, $1=$ Male $)$, and educational status $(0=$ Completed statutory education, $1=$ Did not complete statutory education).

To test for the unique and combined effect of childhood sexual and physical abuse, a second set of five binary logistic regression analyses were conducted with the same outcome variables as in the previous analyses. For each analysis three predictors were included; childhood sexual abuse ( $1=$ Yes, $0=$ No), childhood physical abuse $(1=$ Yes, $0=$ No), and the interaction between these variables. The interaction term was used to test if the combined effect of childhood sexual and physical abuse added additional risk above and beyond the effect of the individual forms of abuse.

\section{Results}

\subsection{Psychiatric diagnoses}

All participants in the current study received a primary psychiatric diagnosis according to the ICD-10 codes. Psychotic-based disorders were most common $(86.3 \%, \mathrm{n}=374)$, with schizophrenia (F20) being the most frequently recorded primary diagnosis $(70.0 \%, \mathrm{n}=303)$. Table 1 provides a full description of all primary diagnoses.

\subsection{Childhood adversity}

The frequencies, and gender differences, for each childhood adversity experience are reported in Table 2 . The majority of respondents indicated exposure to at least one adverse event during childhood $(80.4 \%, \mathrm{n}=275)$, and the mean number of experiences was $2.53(M d n=2.0, \mathrm{SD}=2.21$, Range $0-12)$. There was no difference between males and females in relation to the mean number of adversities ( $\mathrm{t}(349)=.94, \mathrm{p}=.346$, Cohen's $\mathrm{d}=.15$ ). The most commonly reported experience was physical abuse (40.1\%, $\mathrm{n}=145)$. Experiences reflecting a disrupted and chaotic home life were frequently indicated and these included one parent leaving the family home $(31.8 \%, \mathrm{n}=134)$, parental misuse of drugs or alcohol $(25.1 \%, \mathrm{n}=106)$, childhood sexual abuse $(22.8 \%$, $\mathrm{n}=81)$, being bullied $(21.3 \%, \mathrm{n}=90)$, and changing schools $(20.9 \%, \mathrm{n}=88)$. Females were significantly more likely than males to have been bullied $(\chi 2(1)=16.88, p<.001$, OR $=3.32)$, and raised by a parent with an extended period of unemployment $(\chi 2$ $(1)=6.15, \mathrm{p}=.013$, OR $=2.94)$.

\subsection{Childhood adversity as a predictor of criminal and psychiatric outcomes}

As shown in Table 3, childhood adversity was significantly associated with an increased risk of prior criminal convictions $(\mathrm{OR}=1.26)$, engagement in animal abuse $(\mathrm{OR}=1.45)$, suicidal and self-injurious behaviour $(\mathrm{OR}=1.21)$, and problematic drug or alcohol use $(\mathrm{OR}=1.23)$. In terms of the covariates, males were significantly more likely to have a prior criminal conviction $(\mathrm{OR}=4.93)$ and problematic drug or alcohol usage $(\mathrm{OR}=4.62)$, and were significantly less likely to have engaged in suicidal and self-injurious behaviour ( $\mathrm{OR}=.32$ ). Not finishing school was significantly associated with an increased risk of having a prior criminal conviction ( $\mathrm{OR}=3.32$ ), engagement in suicidal and self-injurious behaviour $(\mathrm{OR}=1.81$ ), and problematic drug or alcohol use 
Table 1

Prevalence of ICD10 psychiatric diagnoses $(\mathrm{N}=422)$.

\begin{tabular}{lll}
\hline & $\mathrm{N}$ & $\%$ \\
\hline F10.1 Alcohol Harm & 1 & .2 \\
F19.1 Drug Harm & 1 & .2 \\
F19.2 Drug Dependence & 1 & .2 \\
F15.3/19.3 Drug Withdrawal & 4 & .9 \\
F20 Schizophrenia & 303 & 71.8 \\
F21 Schizotypal & 1 & .2 \\
F22 Persistent Delusional Disorder & 4 & .9 \\
F23 Acute Transient Psychotic Disorder & 1 & .2 \\
F24 Drug Psychosis & 1 & .2 \\
F25 Schizoaffective Disorder & 34 & 8.1 \\
F29 Unspecified Non-Organic Psychosis & 4 & .9 \\
F30 Manic Episode & 1 & .2 \\
F31 Bipolar Affective Disorder & 25 & 5.9 \\
F32 Depressive Episode & 2 & .5 \\
F33 Recurrent Depressive Disorder & 1 & .2 \\
F41 Anxiety Disorder & 0 & 0 \\
F43.1 Posttraumatic Stress Disorder & 2 & .5 \\
F43.2 Adjustment Disorder & 0 & 0 \\
F60.2 Antisocial Personality Disorder & 10 & 2.4 \\
F60.3 Emotionally Unstable Personality Disorder & 10 & 2.4 \\
F60.4 Histrionic Personality Disorder & 0 & 0 \\
F60.5 Anakastic Personality Disorder of OCD & 0 & 0 \\
F60.6 Anxious-Avoidant Personality Disorder & 0 & 0 \\
F60.7 Dependent Personality Disorder & 0 & 0 \\
F61 Mixed Personality Disorder & 5 & 1.2 \\
F60.81 Narcissistic Personality Disorder & 0 & 0 \\
F84 Autistic Spectrum Disorder & 0 & 0 \\
F84.5 Asperger's Syndrome & 0 & 0 \\
F90 Attention Deficit Hyperactivity Disorder & 2 & .5 \\
F91 Conduct Disorder in Childhood & 0 & 0 \\
Other & 6 & 1.4 \\
Unknown & 3 & .7 \\
\hline & & \\
& 2 & \\
& &
\end{tabular}

Table 2

Cross-tabulations of sex differences on each form of childhood trauma and adversity $(\mathrm{N}=422)$.

\begin{tabular}{|c|c|c|c|c|}
\hline Trauma and Adversity Experiences & $\begin{array}{l}\%(\mathrm{n}) \\
\text { Total Sample }\end{array}$ & $\begin{array}{l}\%(\mathrm{n}) \\
\text { Males }\end{array}$ & $\begin{array}{l}\%(\mathrm{n}) \\
\text { Females }\end{array}$ & OR $(95 \% \mathrm{CI})$ \\
\hline Physical Abuse & $40.1(145)$ & $38.6(127)$ & $54.5(18)$ & $1.91(.93,3.92)$ \\
\hline Sexual Abuse & $22.8(81)$ & $21.5(69)$ & $34.3(12)$ & $1.91(.90,4.02)$ \\
\hline Parental Serious Illness or Accident & $5.0(21)$ & $4.7(18)$ & $7.9(3)$ & $1.74(.49,6.21)$ \\
\hline Sibling Serious Illness or Accident & $1.4(6)$ & $1.6(6)$ & $.0(0)$ & - \\
\hline Death of a Parent & $9.5(40)$ & $9.6(37)$ & $7.9(3)$ & $1.24(.37,4.24)$ \\
\hline Death of a Sibling & $2.8(12)$ & $2.6(10)$ & $5.3(2)$ & $2.08(.44,9.85)$ \\
\hline Death of Grandparent or Close Relative & $2.6(11)$ & $2.6(10)$ & $2.6(1)$ & $1.01(.13,8.12)$ \\
\hline Long-term Parental Unemployment & $6.4(27)$ & $5.5(21)$ & $15.8(6)$ & $3.24(1.22,8.61)^{*}$ \\
\hline Another Child Stopped Living at Home & $1.7(7)$ & $1.8(7)$ & $.0(0)$ & - \\
\hline Another Child Moved into Home & $1.2(5)$ & $1.3(5)$ & $.0(0)$ & - \\
\hline Lived in Temporary Accommodation & $3.6(15)$ & $3.1(12)$ & $7.9(3)$ & $2.66(.72,9.87)$ \\
\hline Extended Parental Absence & $9.2(39)$ & $8.6(33)$ & $15.8(6)$ & $1.99(.78,5.12)$ \\
\hline New Adult Entered Home & $10.9(46)$ & $10.9(42)$ & $10.5(4)$ & $1.04(.35,3.09)$ \\
\hline Parental Drug/Alcohol Misuse & $25.1(106)$ & 24.7 (95) & $28.9(11)$ & $1.24(.59,2.59)$ \\
\hline Parent Left Home & $31.8(134)$ & $32.8(126)$ & $21.1(8)$ & $1.83(.82,4.11)$ \\
\hline Moved House & $17.5(74)$ & $17.2(66)$ & $21.1(8)$ & $1.29(.56,2.93)$ \\
\hline Moved in State Care & $16.4(69)$ & $16.7(64)$ & $13.2(5)$ & $1.32(.50,3.51)$ \\
\hline Changed School & $20.9(88)$ & $21.1(81)$ & $18.4(7)$ & $1.18(.50,2.79)$ \\
\hline Bullied in School & $21.3(90)$ & $18.8(72)$ & $47.4(18)$ & $3.90(1.96,7.75)$ \\
\hline
\end{tabular}

Note: OR $(95 \% \mathrm{CI})=$ Odds Ratio with 95\% Confidence Intervals.

$* p<.05$.

$(\mathrm{OR}=2.18)$.

The majority of the sample reported experiencing neither childhood sexual or physical abuse (57.3\%); the experience of both sexual and physical abuse (16.7\%) was much more common than sexual abuse only (5.3\%), and $20.8 \%$ reported only physical abuse. The binary logistic regression models that used sexual abuse, physical abuse, and the sexual abuse/physical abuse interactions were 
Table 3

Binary Logistic Regression analyses predicting multiple criminal and psychiatric outcomes.

\begin{tabular}{|c|c|c|c|c|c|c|c|c|c|c|}
\hline & \multicolumn{2}{|c|}{ Prior Criminal Convictions } & \multicolumn{2}{|c|}{ History of Animal Abuse } & \multicolumn{2}{|c|}{$\begin{array}{l}\text { Prior Psychiatric } \\
\text { Admissions }\end{array}$} & \multicolumn{2}{|c|}{$\begin{array}{l}\text { Suicidal and Self- } \\
\text { Harming Behaviour }\end{array}$} & \multicolumn{2}{|c|}{$\begin{array}{l}\text { Problematic Drug or } \\
\text { Alcohol Usage }\end{array}$} \\
\hline & OR & $95 \% \mathrm{CI}$ & OR & $95 \% \mathrm{CI}$ & OR & $95 \% \mathrm{CI}$ & OR & $95 \% \mathrm{CI}$ & OR & $95 \% \mathrm{CI}$ \\
\hline Age & .99 & $.96-1.02$ & 1.03 & $.97-1.08$ & .98 & $.95-1.01$ & .99 & $.97-1.01$ & .98 & $.95-1.01$ \\
\hline Gender (Male) & $4.93^{\text {k* }}$ & $1.82-13.37$ & 2.62 & $.26-26.16$ & .28 & $.04-2.23$ & $.32 *$ & $.10-.99$ & $4.62^{k * k}$ & $1.70-12.51$ \\
\hline $\begin{array}{l}\text { Education (Did not } \\
\text { finish school) }\end{array}$ & $3.32^{* * * *}$ & $1.70-6.46$ & 1.65 & $.44-6.22$ & 1.43 & $.70-2.92$ & $1.81^{*}$ & $1.06-3.07$ & $2.18^{*}$ & $1.05-4.52$ \\
\hline $\begin{array}{c}\text { Childhood Trauma } \\
\text { and Adversity }\end{array}$ & $1.26^{*}$ & $1.05-1.50$ & $1.45^{\text {** }}$ & $1.16-1.82$ & 1.15 & $.96-1.37$ & $1.21^{* * k}$ & $1.06-1.38$ & $1.23^{*}$ & $1.01-1.50$ \\
\hline
\end{tabular}

Note: OR = Odds Ratio; 95\% CI = 95\% Confidence Interval.

$* p<.05$.

$* * p<.01$.

$* * * p<.001$.

not significant for any outcome variable: prior criminal convictions $\left(\chi^{2}(3)=1.59, \mathrm{p}=.66\right)$, history of animal abuse $\left(\chi^{2}(3)=7.76\right.$, $\mathrm{p}=.05)$, prior psychiatric admissions $\left(\chi^{2}(3)=3.75, \mathrm{p}=.29\right)$, suicidal and self-harming behaviour $\left(\chi^{2}(3)=6.17, \mathrm{p}=.10\right)$, and problematic drug or alcohol usage $\left(\chi^{2}(3)=5.60, \mathrm{p}=.13\right)$.

\section{Discussion}

Using census data collected for all forensic inpatients in Scotland, as of November 2013, we were able to conclusively demonstrate that this population is characterised by extremely high rates of psychotic illness and childhood adversity. Almost $90 \%$ of the forensic inpatient population of Scotland had a psychotic illness as their primary diagnosis, with schizophrenia being the most frequent diagnosis (71.8\%). Schizoaffective Disorder (8.1\%) and Bipolar Affective Disorder (5.9\%) were the next most common primary diagnoses. In addition to these psychiatric disorders, nearly $90 \%$ of inpatients also were reported to have problems with drug or alcohol use, and two-thirds had a history of engaging in suicidal and self-injurious behaviour. Current findings based on census data therefore align with clinical data obtained from smaller, opportunistic samples of forensic inpatients which have indicated a high prevalence of psychotic and substance abuse disorders (Corbett et al., 2018; Kivimies et al., 2012). It was also found that exposure to adverse life events in childhood was common amongst this population of forensic inpatients with $79.2 \%$ having experienced at least one of the 19 experiences that were assessed. A significant proportion of inpatients reported severe traumatic experiences, such as physical and sexual abuse, however, it is important to note that less extreme events such as changing schools, parental divorce, parental drug or alcohol use, moving house frequently, and being taken into state care were also commonly observed. In addition to severe mental illness, forensic inpatients in Scotland are characterised by high levels of childhood adversity, especially events that reflect a chaotic and unstable home life.

The results of the initial binary logistic regression analyses showed that the accumulation of different childhood adversities was a significant predictor of both criminal and psychiatric related outcomes. Results showed that for every additional adverse life event that a person experienced in childhood, the risk of criminal conviction, abuse of animals, suicidal and self-injurious behaviour, and problematic alcohol or drug use increased by between $21 \%$ and $45 \%$, controlling for important risk factors such as age, gender, and educational failure. Consistent with a large body of evidence from the wider prisoner population, our results showed that male forensic inpatients were substantially more likely to have prior criminal convictions (e.g. Moffitt, Caspi, Rutter, \& Silva, 2001) to have problematic drug or alcohol use, and be less likely to have engaged in suicidal and self-injurious behaviour. Moreover, failure to finish school was also associated with an elevated risk of prior criminal convictions, suicidal and self-injurious behaviour, and problematic drug or alcohol use. These results indicate that while childhood adversity plays an important role in understanding the development of dysfunctional psychological and criminal outcomes, demographic and social-developmental factors are also important. The second set of binary logistic regression analyses aimed to assess the specific risk associated with childhood sexual and physical abuse (and their combined effect). None of these models were statistically significant and this indicates that these do not have a unique effect when isolated from the broader set of adversities. This is consistent with previous research that has demonstrated that the cumulative effect of childhood adversity is important in predicting a broad range of negative outcomes such as high-risk behaviour in adolescence (Layne et al., 2014), suicidality (Björkenstam, Hjern, Björkenstam, \& Kosidou, 2018), poor physical health (Hughes et al., 2017), and personality disorders (Björkenstam, Ekselius, Burström, Kosidou, \& Björkenstam, 2017). Indeed, a metaanalysis of studies examining the relationship between childhood trauma and psychosis reported a significant dose-response relationship, increasing levels of childhood trauma exposure was associated with an increased risk of reporting psychotic symptoms or disorder (Varese et al., 2012).

Individuals with a history of imprisonment, and psychiatric disorders such as schizophrenia, are at a greater risk of early mortality. A population-based study in Finland showed a reduced life expectancy of between 17-22 years for those diagnosed with schizophrenia (Tiihonen et al., 2009), while prisoners are up to five times more likely to commit suicide (Fazel, Grann, Kling, \& Hawton, 2011), and 29 times more like to die within the first week of release from prison relative to the general population (Binswanger et al., 2007). Several studies have shown that forensic inpatients characterised by both of these risk factors for early 
mortality are at a greater risk of early death (Björk \& Lindqvist, 2005; Reiter, 1974). In a sample of 1253 Finnish forensic inpatients followed over a 15-year period, those individuals detained in a forensic setting were three times more likely than the general population to have died (Ojansuu, Putkonen, \& Tiihonen, 2015). Effective management of forensic inpatients, given their highly vulnerable status, is therefore essential. However, a survey of the English prison system found that only one-quarter of severely mentally ill prisoners were ever assessed, and only 13\% ever received any form of treatment (Senior et al., 2013).

Developing a sophisticated understanding of the role of childhood adversity amongst forensic inpatients, and the psychological mechanisms that lead to psychotic symptoms and criminal activity may assist with developing interventions that promote recovery (Brand, Rossell, Bendall, \& Thomas, 2017)· A number of theoretical frameworks have attempted to explain the relationship between childhood trauma and psychosis. For example, it has been proposed that traumatic stress and psychosis involve shared mechanisms such as dissociation, attributional style, and interpretation of intrusions (Bendall et al., 2013). Posttraumatic intrusions may be misattributed by individuals to an external source and subsequently experienced as hallucinations. Another theory has proposed that childhood adversity is associated with both an externalising attribution style and a tendency to perceive the world as hostile (Bentall \& Fernyhough, 2008). Exposure to childhood adversity may lead to negative cognitive schemas about oneself and others; disruption of normative mechanisms of coping with stress and interpersonal frustration; and facilitate the development of maladaptive coping strategies, all of which could contribute to the deleterious psychological and behavioural consequences observed amongst forensic inpatients.

Thus, we recommend the provision of trauma-informed care (e.g. Miller \& Najavits, 2012) within forensic settings in which programs take active steps to create safe environments that model healthy boundaries and non-threatening interactions. Forensic units should provide opportunities to learn and rehearse self-regulation skills, self-reflection, and self-correction. Although there is now an emerging evidence of psychotherapies better suited to states associated with multiple childhood trauma (Cloitre et al., 2010; Matulis, Resick, Rosner, \& Steil, 2014), future research should evaluate the acceptability and efficacy of such interventions in traumatised individuals in secure forensic settings. Similarly, the identification of repeat and serious violent offenders with complex levels of trauma and mental health difficulties within the prison system is an important extension to such work (Mahoney \& Karatzias, 2012). Indeed, early identification and intervention with young people who have histories of childhood trauma is required to improve outcomes and minimize the risk for criminal behavior (Moretti, Bartolo, Craig, Slaney, \& Odgers, 2014) as well as recidivism.

Although the census level data is a key strength of this study, the nature of the data collection did result in some limitations. For example, only data on primary psychiatric diagnoses were collected. It is highly probable that this population experienced a range of comorbid diagnoses however it was not possible to ascertain if this was the case given the manner in which the data were collected. It was notable that, collectively, only $1.5 \%$ of individuals had a drug or alcohol related primary diagnosis, despite the fact that $86 \%$ were identified as possessing a history of problematic drug or alcohol use. It is highly probable therefore, that substance abuse disorders were highly comorbid with psychotic disorders within this population. With respect to the criminal behaviour related outcomes, no data was collected about the nature of one's criminal activity and therefore it was not possible to delineate between different forms of criminal behaviour (e.g., violent or non-violent) and their potential association with childhood adversity. Similarly, only basic demographic data was collected on inpatients and as such we were restricted in the number of covariates that could be included within our predictive analysis. Finally, it is also important to highlight females were underrepresented in the sample.

This study marks the first attempt to determine the types of psychiatric disorders, as well as childhood adversity experiences that characterise detained inpatients using census level data. Current results indicate that individuals detained within forensic settings in Scotland are characterised by a history of childhood adversity and maltreatment, psychotic illness, drug and alcohol abuse, and suicidal and self-injurious behaviour. Moreover, one's history of childhood adversity is a robust risk factor for an increased likelihood of multiple deleterious psychiatric and criminal outcomes. These findings highlight the importance of considering history of childhood adversity for forensic inpatients, and suggests that trauma-informed models of care may be beneficial for this highly vulnerable and psychologically impaired population.

\section{References}

Avery, L., Hutchinson, D., \& Whitaker, K. (2002). Domestic violence and intergenerational rates of child sexual abuse: A case record analysis. Child \& Adolescent Social Work Journal, 19(1), 77-90.

Basto-Pereira, M., Miranda, A., Ribeiro, S., \& Maia, A. (2016). Growing up with adversity: From juvenile justice involvement to criminal persistence and psychosocial problems in young adulthood. Child Abuse \& Neglect, 62, 63-75. https://doi.org/10.1016/j.chiabu.2016.10.011.

Bendall, S., Hulbert, C. A., Alvarez-Jimenez, M., Allott, K., McGorry, P. D., \& Jackson, H. J. (2013). Testing a model of the relationship between childhood sexual abuse and psychosis in a first-episode psychosis group: The role of hallucinations and delusions, posttraumatic intrusions, and selective attention. Journal of Nervous and Mental Diseases, 201(11), 941-947. https://doi.org/10.1097/NMD.0000000000000033.

Bentall, R. P., \& Fernyhough, C. (2008). Social predictors of psychotic experiences: Specificity and psychological mechanisms. Schizophrenia Bulletin, 34(6), 1012-1020. https://doi.org/10.1093/schbul/sbn103.

Bentall, R. P., Wickham, S., Shevlin, M., \& Varese, F. (2012). Do specific early life adversities lead to specific symptoms of psychosis? A study from the 2007 Adult Psychiatric Morbidity Survey. Schizophrenia Bulletin, 38(4), 734-740. https://doi.org/10.1093/schbul/sbs049.

Binswanger, I. A., Stern, M. F., Deyo, R. A., Heagerty, P. J., Cheadle, A., Elmore, J. G., \& Koepsell, T. D. (2007). Release from prison-A high risk of death for former inmates. New England Journal of Medicine, 356(2), 157-165. https://doi.org/10.1056/NEJMsa064115.

Birmingham, L., Mason, D., \& Grubin, D. (1996). Prevalence of mental disorder in remand prisoners: Consecutive case study. British Medical Journal, 313(7071), 1521-1524. https://doi.org/10.1136/bmj.313.7071.1521.

Björk, T., \& Lindqvist, P. (2005). Mortality among mentally disordered offenders: A community based follow-up study. Criminal Behaviour and Mental Health, 15(2), 93-96. https://doi.org/10.1002/cbm.41.

Björkenstam, E., Ekselius, L., Burström, B., Kosidou, K., \& Björkenstam, C. (2017). Association between childhood adversity and a diagnosis of personality disorder in young adulthood: A cohort study of 107,287 individuals in Stockholm County. European Journal of Epidemiology, 32(8), 721-731.

Björkenstam, E., Hjern, A., Björkenstam, C., \& Kosidou, K. (2018). Association of cumulative childhood adversity and adolescent violent offending with suicide in early 
adulthood. JAMA Psychiatry, 75(2), 185-193. https://doi.org/10.1001/jamapsychiatry.2017.3788.

Brand, R. M., Rossell, S. L., Bendall, S., \& Thomas, N. (2017). Can we use an interventionist casual paradigm to untangle the relationship between trauma, PTSD and psychosis? Frontiers in Psychology, 306. https://doi.org/10.3389/fpsyg.2017.00306.

Cloitre, M., Stovall-McClough, K. C., Nooner, K., Zorbas, P., Cherry, S., Jackson, C. L., ... Petkova, E. (2010). Treatment for PTSD related to childhood abuse: A randomized controlled trial. American Journal of Psychiatry, 167(8), 915-924. https://doi.org/10.1176/appi.ajp.2010.09081247.

Corbett, L., Karyad, K. A., Kinney, D., Nitch, S. R., Bayan, S. M., \& Williams, M. (2018). Impaired verbal learning in forensic inpatients with Schizophrenia Spectrum Disorder. Applied Neuropsychology: Adult, 25(3), 189-196. https://doi.org/10.1080/23279095.2016.1269010.

de Vogel, V., \& Nicholls, T. L. (2016). Gender matters: An introduction to the special issue on women and girls. International Journal of Forensic Mental Health, 15(1), 1-25. https://doi.org/10.1080/14999013.2016.1141439.

Dudeck, M., Sosic-Vasic, Z., Otte, S., Rasche, K., Leichauer, K., Tippelt, S., ... Streb, J. (2016). The association of adverse childhood experiences and appetitive aggression with suicide attempts and violent crimes in male forensic psychiatry inpatients. Psychiatry Research, 240, 352-357. https://doi.org/10.1016/j.psychres. 2016.04.073.

Fazel, S., \& Seewald, K. (2012). Severe mental illness in 33,588 prisoners worldwide: Systematic review and meta-regression analysis. British Journal of Psychiatry, 200(5), 364-373. https://doi.org/10.1192/bjp.bp.111.096370.

Fazel, S., Grann, M., Kling, B., \& Hawton, K. (2011). Prison suicide in 12 countries: An ecological study of 861 suicides during 2003-2007. Social Psychiatry and Psychiatric Epidemiology, 46(3), 191-195. https://doi.org/10.1007/s00127-010-0184-4.

Hughes, K., Bellis, M. A., Hardcastle, K. A., Sethi, D., Butchart, A., Mikton, C., Jones, L., \& Dunne, M. P. (2017). The effect of multiple adverse childhood experiences on health: A systematic review and meta-analysis. The Lancet Public Health, 2(8), e356-e366.

Jonas, S., Bebbington, P., McManus, S., Meltzer, H., Jenkins, R., Kuipers, E., \& Brugha, T. (2011). Sexual abuse and psychiatric disorder in England: Results from the 2007 adult psychiatric morbidity survey. Psychological Medicine, 41(4), 709-719. https://doi.org/10.1017/S003329171000111X.

Jones, L. (2015). The Peaks Unit: From a pilot for 'untreatable psychopaths to trauma informed milieu therapy. Prison Service Journal, 218, 17-23.

Karatzias, T., Power, K., Woolston, C., Apurva, P., Begley, A., Mirza, K., ... Purdie, A. (2018). Multiple traumatic experiences, post-traumatic stress disorder and offending behaviour in female prisoners. Criminal Behaviour and Mental Health, 28(1), 72-84. https://doi.org/10.1002/cbm.2043.

Kivimies, K., Repo-Tiihonen, E., \& Tiihonen, J. (2012). The substance use among forensic psychiatric patients. American Journal of Drug and Alcohol Abuse, 38(4), 273-277. https://doi.org/10.3109/00952990.2011.643972.

Layne, C. M., Greeson, J. K. P., Ostrowski, S. A., Kim, S., Reading, S., Vivrette, R. L., ... Pynoos, R. S. (2014). Cumulative trauma exposure and high risk behavior in adolescence: Findings from the National Child Traumatic Stress Network Core Data Set. Psychological Trauma: Theory, Research, Practice, and Policy, 6(Suppl. 1), S40-S49. https://doi.org/10.1037/a0037799.

Mahoney, A. L., \& Karatzias, T. (2012). Violent female offending: An exploration of repeat and one-time offending. International Journal of Forensic Mental Health, 11, 191-202. https://doi.org/10.1080/14999013.2012.723664.

Matulis, S., Resick, P. A., Rosner, R., \& Steil, R. (2014). Developmentally adapted cognitive processing therapy for adolescents suffering from posttraumatic stress disorder after childhood sexual or physical abuse: A pilot study. Clinical Child and Family Psychology Review, 17(2), 173-190. https://doi.org/10.1007/s10567-0130156-9.

Miller, N. A., \& Najavits, L. M. (2012). Creating trauma-informed correctional care: A balance of goals and environment. European Journal of Psychotraumatology, 3 , 17246. https://doi.org/10.3402/ejpt.v3i0.17246.

Moffitt, T. E., Caspi, A., Rutter, M., \& Silva, P. A. (2001). Sex differences in antisocial behaviour: Conduct disorder, delinquency, and violence in the Dunedin Longitudinal Study. Cambridge University Press.

Moretti, M. M., Bartolo, T., Craig, S., Slaney, K., \& Odgers, C. (2014). Gender and transmission of risk: A prospective study of adolescent girls exposed to maternal versus paternal interparental violence. Journal of Research on Adolescents, 24(1), 80-92. https://doi.org/10.1111/jora.12065.

Ojansuu, I., Putkonen, H., \& Tiihonen, J. (2015). Mortality among forensic psychiatric patients in Finland. Nordic Journal of Psychiatry, 69(1), 25-27. https://doi.org/ $10.3109 / 08039488.2014 .908949$.

Reiter, K. (1974). Mortality and causes of death in men found to be mentally abnormal by forensic psychiatrists. Scandinavian Journal of Social Medicine, 2, 1-3.

Senior, J., Birmingham, L., Harty, M. A., Hassan, L., Hayes, A. J., Kendall, K., ... Shaw, J. (2013). Identification and management of prisoners with severe psychiatric illness by specialist mental health services. Psychological Medicine, 43, 1511-1520. https://doi.org/10.1017/S0033291712002073.

Shevlin, M., Dorahy, M. J., \& Adamson, G. (2007). Trauma and psychosis: an analysis of the national co-morbidity survey. American Journal of Psychiatry, 164(1), 166-169. https://doi.org/10.1176/ajp.2007.164.1.166.

Singleton, N., Meltzer, H., Gatward, R., Coid, J., \& Deasy, D. (1998). Survey of psychiatric morbidity among prisoners in England and Wales. London Department of Health.

Spitzer, C., Chevalier, C., Gillner, M., Freyberger, H. J., \& Barlow, S. (2006). Complex posttraumatic stress disorder and child maltreatment in forensic inpatients. The Journal of Forensic Psychiatry \& Psychology, 17(2), 204-216. https://doi.org/10.1080/14789940500497743.

Tiihonen, J., Lönnqvist, J., Wahlbeck, K., Klaukka, T., Niskanen, L., Tanskanen, A., \& Haukka, J. (2009). 11 year follow-up of mortality in patients with schizophrenia: A population-based cohort study (FIN11 study). Lancet, 374(9690), 620-627. https://doi.org/10.1016/S0140-6736(09)60742-X.

Varese, F., Smeets, F., Drukker, M., Lieverse, R., Lataster, T., Viechtbauer, W., Read, J., Van Os, J., \& Bentall, R. P. (2012). Childhood adversities increase the risk of psychosis: A meta-analysis of patient-control, prospective-and cross-sectional cohort studies. Schizophrenia Bulletin, 38(4), 661-671. 